\title{
Epidural hematoma in patients with ankylosing spondylitis requiring surgical stabilization: a single-institution retrospective review with literature analysis
}

\author{
George Hanna, MD, ${ }^{1}$ Syed-Abdullah Uddin, BS, ${ }^{1,3}$ Andrew Trontis, MD, ${ }^{2}$ Lindsey Ross, MD, ${ }^{1}$ \\ Doniel Drazin, MD, ${ }^{4}$ Terrence T. Kim, MD, ${ }^{2}$ and J. Patrick Johnson, MD ${ }^{1}$
}

1Department of Neurological Surgery, Cedars-Sinai Medical Center, Los Angeles; '2Department of Orthopaedics, Cedars-Sinai Medical Center, Los Angeles; and 'University of California, Riverside (UCR) School of Medicine, Riverside, California; and ${ }^{4}$ Pacific Northwest University of Health Sciences College of Medicine, Yakima, Washington

\begin{abstract}
OBJECTIVE Ankylosing spondylitis (AS) is a chronic inflammatory disease affecting the sacroiliac joints and axial spine that is closely linked with human leukocyte antigen-B27. There appears to be an increased frequency of associated epidural hematomas in spine fractures in patients with AS. The objective was to review the incidence within the literature and a single-institution experience of the occurrence of epidural hematoma in the context of patients with AS requiring spine surgery.
\end{abstract}

METHODS Deep 6 Al software was used to search the entire database of patients at a single level I trauma center (since the advent of the institution's modern electronic health record system) to look at all patients with AS who underwent spinal surgery and who had a diagnosis of epidural hematoma. Additionally, a systemic literature review was performed of all papers evaluating the incidence of epidural hematoma in patients with spine fractures.

RESULTS A single-institution, retrospective review of records from 2009 to 2020 yielded a total of 164 patients with AS who underwent spine surgery. Of those patients, 17 (10.4\%) had epidural hematomas on imaging, with the majority requiring surgical decompression. These spine fractures occurred close to the cervicothoracic or thoracolumbar junction. The patients ranged in age from 51 to 88 years, and there were 14 males and 3 females in the cohort. Eight patients were administered an antiplatelet and/or anticoagulant agent, and the rest were not. All patients required surgical stabilization, with $64.7 \%$ of patients also requiring decompressive laminectomies for evacuation of the hematoma and spinal cord decompression. Only 1 death was reported in the series. There was a tendency toward neurological improvement after surgical intervention.

CONCLUSIONS AS has been a well-described pathologic process that leads to an increased risk of three-column injury in spine fracture, with an increased incidence of symptomatic epidural hematoma compared with patients without AS. Early recognition of this entity is important to ensure that appropriate surgical management includes addressing compression of the neural elements in addition to surgical stabilization.

https://thejns.org/doi/abs/10.3171/2021.7.FOCUS21334

KEYWORDS ankylosing spondylitis; spine surgery; spine fusion; extradural hematoma; EDH; spinal epidural hematoma

A NKYLOSING spondylitis (AS) is a chronic inflammatory disease of the sacroiliac and axial joints with multiple extraspinal manifestations that is closely linked with the presence of human leukocyte antigen-B27 (HLA-B27). ${ }^{1}$ Syndesmophyte formation with decreased motion and spinal deformity can lead to significant dis- ability. While exercise and continued motion remain the mainstay of treatment, recent pharmacological advancements and the use of nonsteroidal antiinflammatory drugs (NSAIDs), antitumor necrosis factor agents, and infliximab have significantly improved functional outcomes. ${ }^{2}$ More men than women are affected, and the incidence

ABBREVIATIONS AS = ankylosing spondylitis; $\mathrm{EDH}=$ epidural hematoma .

SUBMITTED June 2, 2021. ACCEPTED July 16, 2021.

INCLUDE WHEN CITING DOI: 10.3171/2021.7.FOCUS21334. 
ranges from 0.5 to 14 per 100,000 members of the population. ${ }^{3}$

\section{Spine Fractures in AS}

Patients with AS, as in other chronic inflammatory rheumatic diseases, are at higher risk of low bone mass, which predisposes them to fractures. ${ }^{4} \mathrm{~A}$ recent review identified that low-frequency impacts in patients with AS, such as ground-level falls or trivial traumas, can lead to a significant compromise of spinal stability requiring CT and MRI for evaluation and often necessitating surgical stabilization. ${ }^{5}$ Chronic inflammation, bone remodeling, and osseous resorption lead to osteoporosis and a more rigid spine that predisposes patients to increased fractures that can often be detected on CT; however, occult fractures can be missed and need to be evaluated on MRI. ${ }^{6}$

\section{Spinal EDH and AS}

One of the earliest descriptions of surgical intervention required in a patient with AS found to have an epidural hematoma (EDH), after a ground-level fall during a generalized tonic-clonic seizure, was in 1979.7 A recent radiographic study looking at patients with urgent and emergency MRI to evaluate spine trauma identified a rate of $68 \%$ of patients with AS who had EDHs. ${ }^{8}$ While the relationship between spinal EDHs in patients with AS and traumatic spine fractures has been described in previous publications, ${ }^{9-12}$ there has certainly been a paucity of literature describing this important relationship that can have dire neurological consequences if not recognized early. ${ }^{13}$ To further evaluate and describe the incidence of this relationship, we have reviewed our institutional experience and the published literature.

\section{Methods}

\section{Retrospective Cohort Analysis}

Using Deep 6 AI software, we performed an artificial intelligence-driven search (see below for detailed explanation) analyzing a complete database of patients at our level I trauma and comprehensive stroke medical center since the incorporation of Epic (Epic Systems Corp.) electronic health record software in 2009. Using search terms to find all patients diagnosed with AS, we found 10,000 patients, of whom 164 were verified to have spine fractures that were managed with surgical intervention. We then queried the records of those patients for a diagnosis of EDH and found the 17 patients included in our study; $10.4 \%$ of patients (17/164) with AS with spine fractures also had a spinal EDH. We then critically analyzed and performed retrospective chart reviews of all patients within this cohort.

\section{Deep 6 Al Search Platform}

According to the company and the explanation of their software, the Deep 6 AI search platform uses a variety of tools and techniques to perform its precision matching rather than relying on a single algorithm. First, clinical concepts are parsed from a wide range of clinical data sources that are both structured and unstructured. These sources are updated nightly to offer the most recent results. Next, 100 different clinical ontologies are combined to accurately recognize clinical concepts from unstructured or semistructured data sources, such as progress or consultation notes. A set of annotators then parses all source data, and labels and stores clinical concepts in a graph database. This turns different clinical notes or fields into a multidimensional vector that can represent a patient and then become a node in the network. Other machine learning routines help disambiguate concepts, detect negations and hypotheticals, and reconcile different concepts and rank results based on a multifactor confidence score. The user can then write a query that searches against indexed patient graphs and retrieves results across millions of patients in a few seconds. The way the queries are constructed determines the precision and recall, and the Deep 6 AI platform favors recalls that allow for queries to return any matching patient according to the criteria of the original query, even if a single concept is selected, such as "ankylosing spondylitis" in our search. The patient evidence timeline makes it simple and quick to validate those matched patients by presenting the source data in a single place for easy review. Finally, validation then informs further filtering for higher-precision queries using additional filters (e.g., time and demographic) and Boolean functions.

\section{Literature Review}

We used the PubMed database to locate articles that identified patients with AS and EDH. We then used the keywords "ankylosing spondylitis" AND "epidural hematoma," which yielded 55 results. Including only Englishlanguage papers, the results were narrowed to 49 . We also excluded papers in which traumatic etiologies with spinal fractures were not the cause of the EDH. One paper was excluded because it was unclear whether the case reported ${ }^{14}$ was previously discussed in the case series by the same lead author years prior. ${ }^{15}$ Two separate authors (G.H. and S.A.U.) independently reviewed each of the papers, and a third author (T.T.K.) reviewed these articles to come to the consensus that 28 articles met the inclusion criteria of being clinical articles that reviewed demographics and outcomes in patients with AS who sustained spinal fractures and were found to have a spinal EDH.

\section{Results}

\section{Patient Demographics and Results}

Our retrospective review of patients with AS requiring spine surgery between 2009 and 2020 revealed 164 patients. Of these patients, 17 had an EDH and required surgical stabilization. There were 14 males and 3 females in our cohort with an age range from 51 to 88 years (average 69.7 years). The predisposing traumatic mechanisms included ground-level falls in 11 patients (Figs. 1 and 2), motor vehicle collision in 3 patients, fall from a height in 1 patient, and unknown etiology in 2 patients. Patient characteristics and demographics are listed in Table 1.

All patients in our cohort required surgical stabilization. Eleven patients (64.7\%) required decompressive laminectomies with evacuation of an EDH, and 1 patient had extensive decompressive laminectomies from C7 to T11 

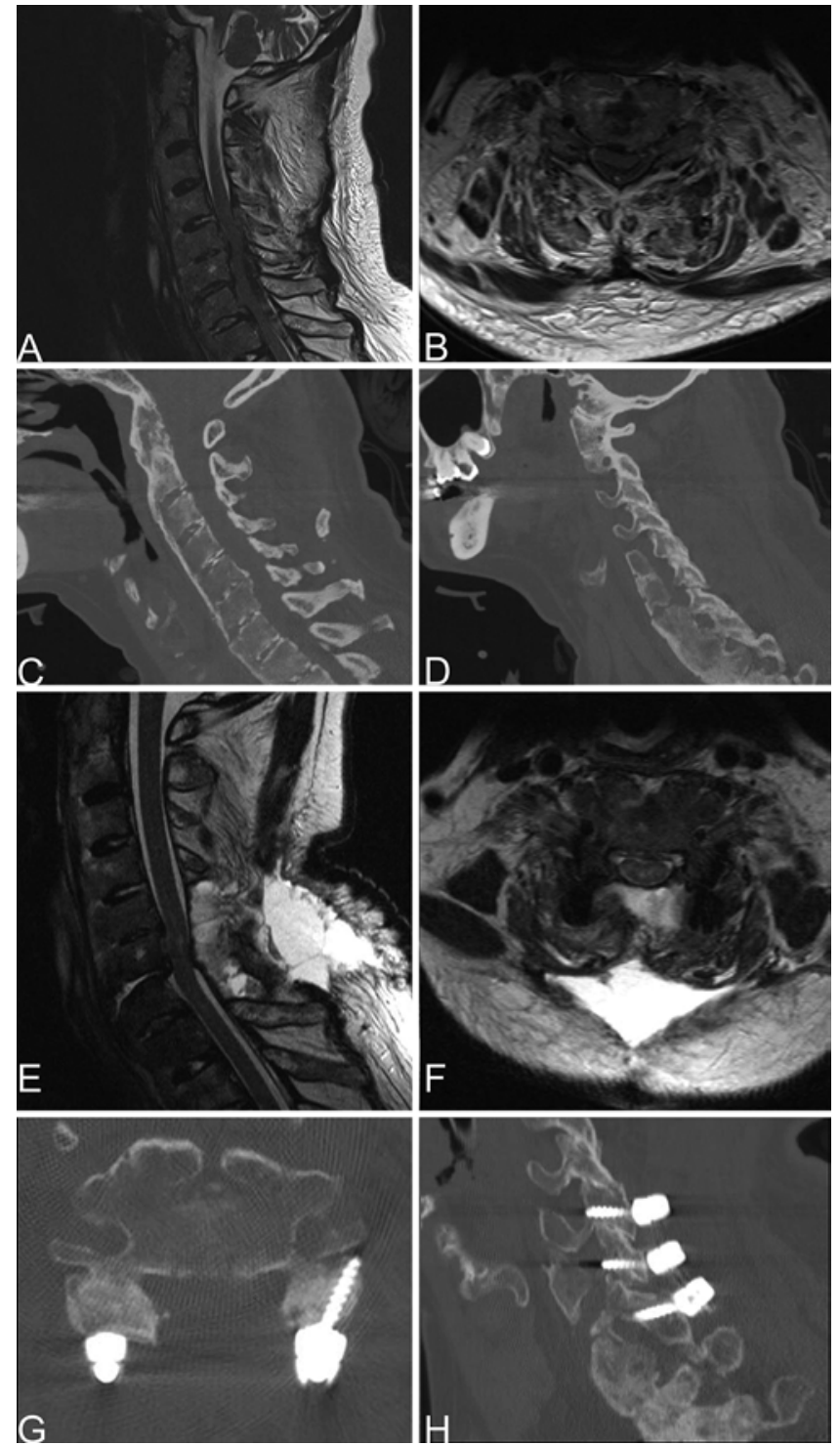

FIG. 1. An 80-year-old female with AS sustained a ground-level fall and was found to have a C5-6 fracture dislocation with a C5-T1 EDH. The patient underwent a $\mathrm{C5}-7$ posterior spinal fusion with laminectomies and evacuation of the EDH. Preoperative T2-weighted sagittal $(\mathbf{A})$ and axial (B) cervical spine MR images demonstrating a C5-T1 EDH. Preoperative sagittal $\mathrm{CT}$ scans of the cervical spine $(\mathbf{C}$ and $\mathbf{D})$ demonstrating a C5-6 fracture dislocation. Postoperative T2-weighted sagittal $(\mathrm{E})$ and axial $(\mathbf{F})$ cervical spine MR images demonstrating decompression and a C5-6 cord signal change. Postoperative axial (G) and sagittal $(\mathbf{H})$ CT scans of the cervical spine, demonstrating wide laminectomies for decompression and $\mathrm{C5}-7$ posterior spinal instrumentation.

at an outside institution, years prior to presentation, for a traumatic EDH (Fig. 3). Eight patients were administered antiplatelet and/or anticoagulant agents, with the majority being on a regimen of $81 \mathrm{mg}$ of aspirin.

The mean blood loss during surgery was $585 \mathrm{ml}$ (range $70-3000 \mathrm{ml}$ ), and the mean surgery duration was 279 minutes (range 119-502 minutes). In $47 \%$ of the surgeries performed, transfusion with one or more blood products or derivatives was used, including packed red blood
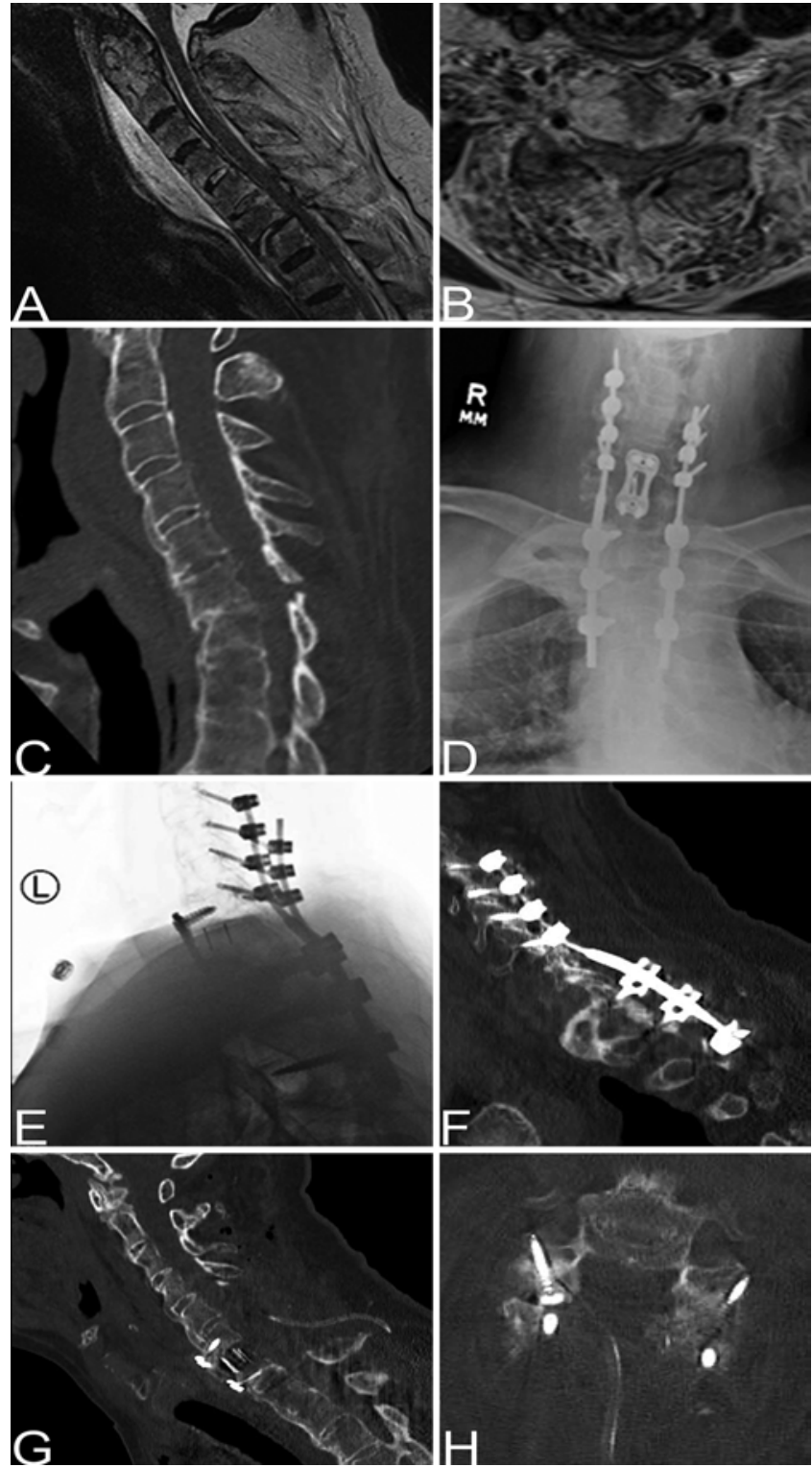

FIG. 2. A 54-year-old male with AS sustained a ground-level fall resulting in severe C3 spinal stenosis, a C6-7 fracture dislocation, and a C5-7 EDH. The patient underwent a C6-7 anterior cervical discectomy and fusion (ACDF), C3-T3 posterior spinal instrumented fusion, and C5-7 laminectomies with evacuation of the EDH. Preoperative T2weighted sagittal (A) and axial (B) cervical spine MR images demonstrating large prevertebral swelling and a C6-7 fracture dislocation with associated EDH and severe stenosis. Preoperative sagittal (C) CT scan of the cervical spine demonstrating a C6-7 fracture dislocation. Postoperative cervical spine anteroposterior (D) and lateral (E) radiographs and a postoperative sagittal $(\mathbf{F})$ CT scan of the cervical spine demonstrating posterior hardware from C3 to T3 and a C6-7 ACDF (G). Postoperative axial CT scan of the cervical spine $(\mathrm{H})$ demonstrating wide laminectomies for decompression at $\mathrm{C} 6$.

cells, fresh frozen plasma, and platelets. The total hospital length of stay was a mean of 20.5 days (range 2-144 days), and if the longest hospital stay outlier was excluded, the mean hospital length of stay was 12.8 days.

We encountered a series of transient complications in 


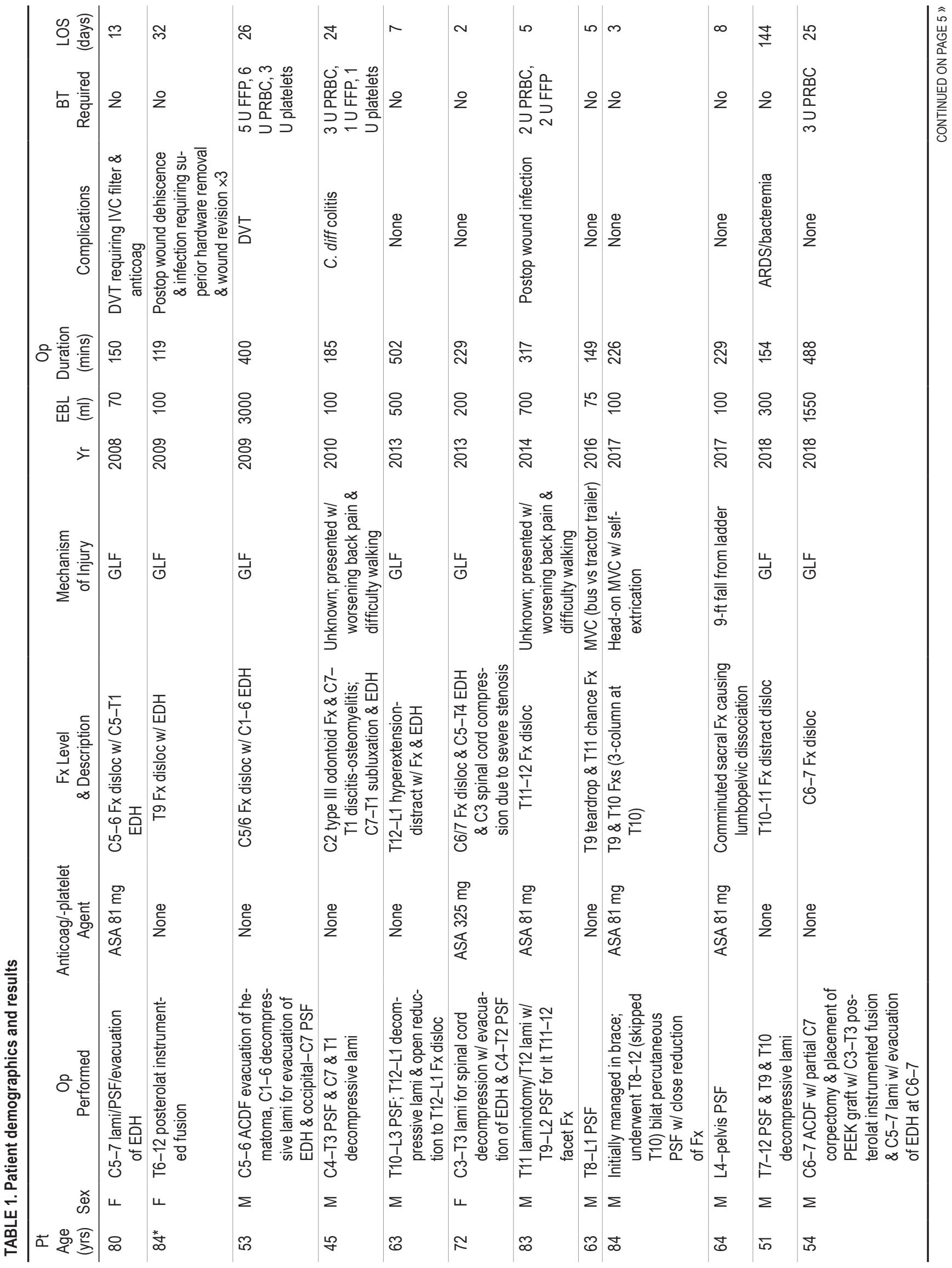




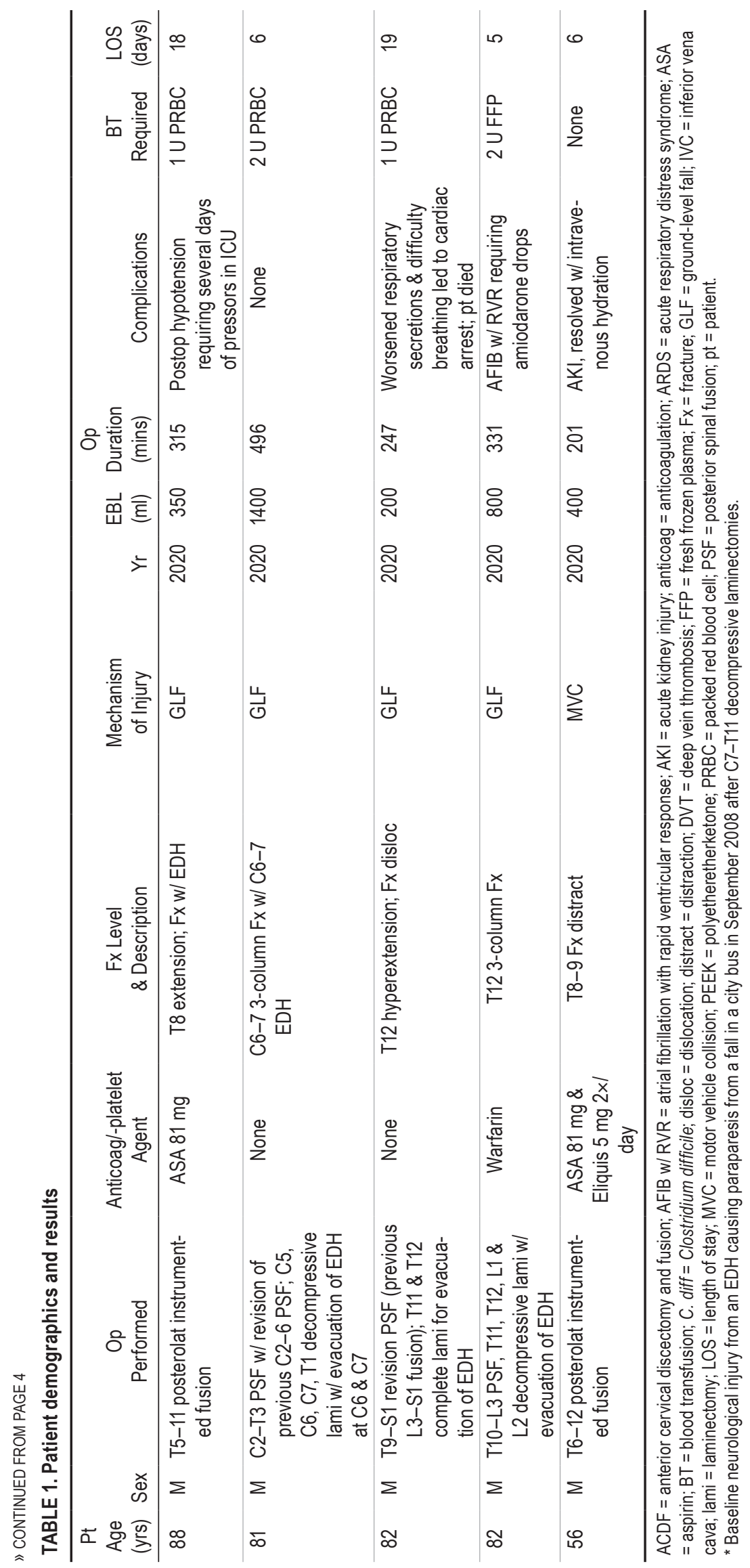

Neurosurg Focus Volume 51 • October 2021 

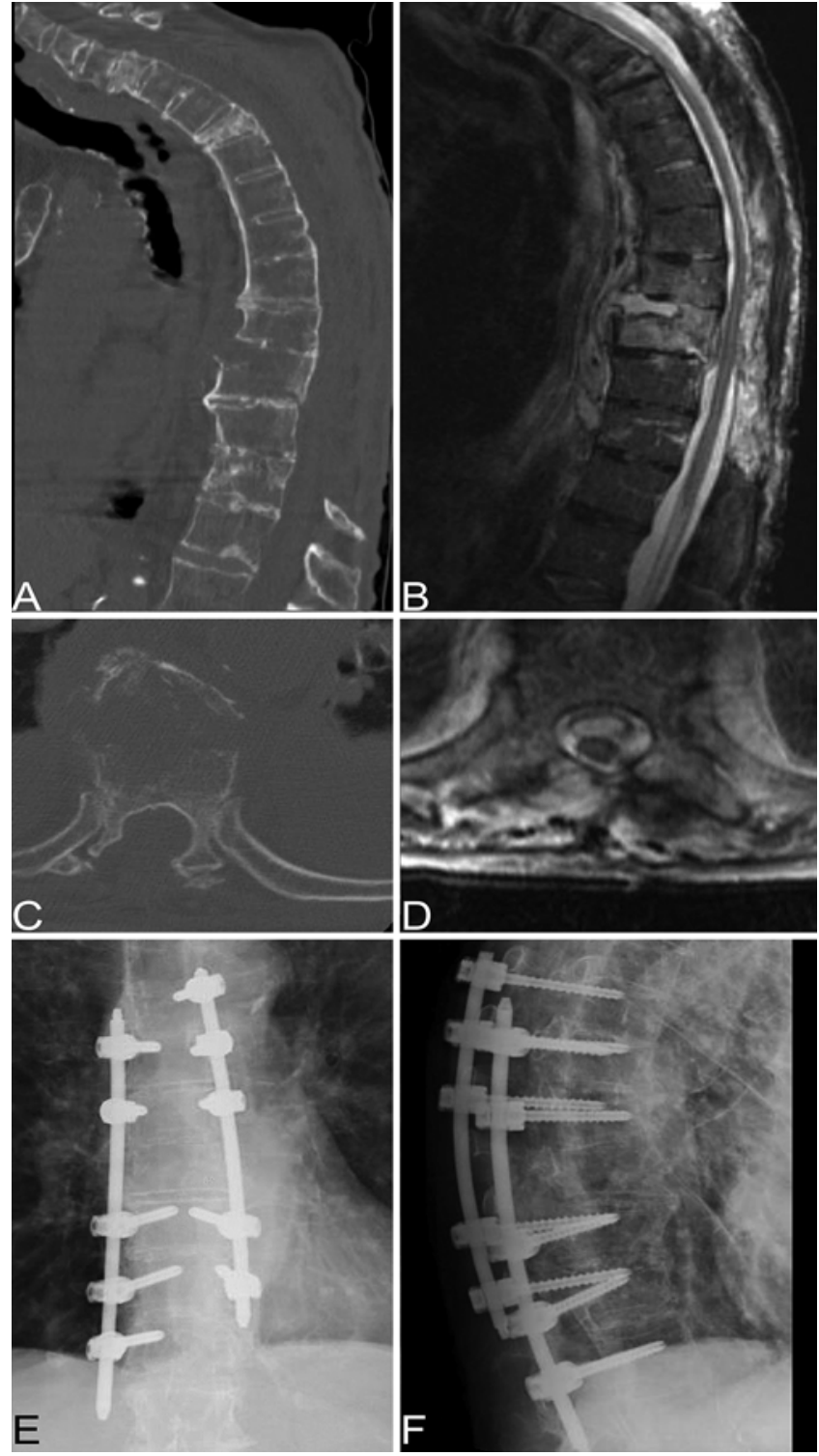

FIG. 3. An 84-year-old female sustained a ground-level fall and presented with T9 fracture dislocation with an associated EDH. The patient underwent T6-12 posterior spinal instrumented fusion. She had previous neurological deficit from a fall on a city bus 1 year prior to presentation and underwent C7-T11 decompressive laminectomies with evacuation of an extensive EDH at that time. Preoperative sagittal (A) and axial (C) CT scans and T2-weighted sagittal (B) and axial (D) MR images of the cervicothoracic spine demonstrating extensive C7-T11 laminectomies and wide decompression with a T9 fracture dislocation and EDH. Postoperative anteroposterior $(\mathbf{E})$ and lateral $(\mathbf{F})$ radiographs demonstrating T6-12 posterior spinal instrumented fusion.

our patients, with only 1 death during hospitalization. Two patients developed deep venous thrombosis that required anticoagulation. The patient with the longest hospital length of stay developed acute respiratory distress syndrome and bacteremia, while the single death in our series occurred later in their hospitalization when the patient developed respiratory failure and subsequent cardiac arrest. Two patients developed postoperative wound infections requiring revision and washout. One patient developed acute kidney injury that resolved with intravenous hydration; and 2 patients required hemodynamic monitoring in the ICU, 1 for hypotension requiring pressors and 1 for amiodarone infusion to treat atrial fibrillation with rapid ventricular response. Finally, 1 patient developed Clostridium difficile colitis during hospitalization. In total, 10 patients $(58.8 \%)$ had an in-hospital complication. There were no patients who had worsening neurological dysfunction, and there was a tendency toward neurological improvement prior to discharge (Table 2).

\section{Literature Review Results and Findings}

Throughout the review of the literature, various mechanisms of trauma were noted, and oftentimes progressive neurological deficit was noted to be associated with the discovery of an EDH concomitant with the spinal fracture. The results of our literature review are summarized in Table 3., $, 8,10,12,15-38$

\section{Discussion}

\section{Mechanism of Injury}

Recorded mechanisms of injury within our cohort and in the literature review yielded a broad range of mechanisms but were often low-force impacts such as a ground-level fall. The earliest reported case in the Englishlanguage literature was in a patient who sustained generalized tonic-clonic seizures and tetraparesis who was found to have an extensive EDH from C6 to T8. ${ }^{7}$ Eleven of our patients $(64.7 \%)$ sustained ground-level falls which caused traumatic fractures and spinal EDH. High-impact mechanisms were also noted, including motor vehicle collisions and fall from a height. These mechanisms were again noted in our literature review. ${ }^{19,31}$

\section{Intervention, Diagnosis, and Outcome}

In our series, we reported that $64.7 \%$, or close to twothirds, of patients required decompressive laminectomies to evacuate the $\mathrm{EDH}$. A recent series of patients with AS demonstrated a $29.3 \%$ incidence of spinal EDH, while the incidence was found to be $19.9 \%$ in patients with and without spinal cord injury in another series..$^{39}$ Other studies also reported a high incidence of EDH after trauma in patients with AS. ${ }^{38,40}$ Most of our patients were diagnosed via MRI or on surgical exploration when neurological deficits were present and we were unable to perform MRI of the area of interest within the spinal axis. Two patients presented without gross neurological deficit and subsequently worsened, while 1 patient had a neurological deficit that improved with surgical immobilization and was found to have an extensive EDH that was evacuated.

Several reports in the literature demonstrated varied presentations, time to diagnosis, and outcomes. A recently published case report demonstrated the first instance of robotic guidance for placement of pedicle screws in addition to decompressive laminectomies ${ }^{37}$ in a patient with $\mathrm{AS}$ and a traumatic spine fracture. In a series of 5 patients, Broom and Raycroft ${ }^{19}$ described a 74-year-old patient with AS with a C5-6 fracture who developed a subacute C6 complete spinal cord injury, even with halo immobilization, and was found to have an EDH that was decom- 
TABLE 2. Neurological function before and after surgical intervention in patients with AS and EDH

\begin{tabular}{|c|c|c|c|}
\hline Pt Age (yrs) & Sex & Presenting Neurological Exam & Neurological Exam Findings at Discharge \\
\hline 80 & $\mathrm{~F}$ & Initially neuro intact; progressed to $\mathrm{C} 4$ complete $\mathrm{SCl}$ & $\begin{array}{l}\text { C7 ASIA grade C SCl; some movement in It UE \& intrinsic w/ } \\
\text { some leg movement; weak triceps on rt, otherwise no move- } \\
\text { ment distally }\end{array}$ \\
\hline $84^{*}$ & $\mathrm{~F}$ & $1-2 / 5$ in proximal LEs \& $3 / 5$ distally (baseline) & Unchanged \\
\hline 53 & M & UEs $2-3 / 5$ \& LEs $0 / 5$ & Unchanged \\
\hline 45 & M & $4 / 5$ It LE \& 4+/5 in It UE & $5 / 5$ in all extremities $w / 4+/ 5$ in bilat iliopsoas \\
\hline 63 & M & Bilat quadriceps \& iliopsoas weakness (4/5) w/ L1 \& L2 numbness & Improved strength \& sensation; ambulates w/ walker \\
\hline 72 & $\mathrm{~F}$ & $\begin{array}{l}\text { Tetraplegia w/ sensation intact; improved to full strength w/ rt UE } \\
0-2 / 5 \text { after collar placed }\end{array}$ & Full strength, except rt hand $2 / 5$ \\
\hline 83 & M & ASIA grade C SCl; 0/5 in EHL/DF/gastrocnemius; $2 / 5$ quadriceps & ASIA grade D SCI; 3/5 DF/PF; 4/5 quadriceps \\
\hline 63 & M & Neuro intact & Neuro intact \\
\hline 84 & M & Neuro intact & Intact \\
\hline 64 & M & Neuro intact & Neuro intact \\
\hline 51 & M & T10 ASIA grade A (complete) paraplegia & T10 ASIA grade A \\
\hline 54 & M & Neuro intact & Neuro intact \\
\hline 88 & M & Neuro intact & Neuro intact \\
\hline 81 & M & Lt UE WE/HG/intrinsic 4/5 & Lt UE improved to $4+/ 5$ \\
\hline 82 & M & Neuro intact & Neuro intact \\
\hline 82 & M & Initially neuro intact; progressed to $1 / 5$ in LEs w/ L2 sensory level & Improved to $4-5 / 5$ in LE \\
\hline 56 & M & Neuro intact & Neuro intact \\
\hline
\end{tabular}

ASIA = American Spine Injury Association impairment scale; $D F=$ dorsiflexion (ankle); EHL = extensor hallucis longus; $H G=$ hand grip; $L E=$ lower extremity; $L L E=$ left lower extremity; neuro = neurologically; $\mathrm{PF}=$ plantar flexion; $\mathrm{SCl}=$ spinal cord injury; $\mathrm{UE}=$ upper extremity; $\mathrm{WE}=$ wrist extension.

* Baseline neurological injury from an EDH causing paraparesis from a fall in a city bus in September 2008 after C7-T11 decompressive laminectomies.

pressed and evacuated, but the patient remained without neurological improvement. In another study, a 91-year-old patient with AS had a delayed diagnosis of a thoracic spine fracture that was initially managed conservatively, but the patient's condition progressed to paraplegia. He was diagnosed with an EDH after neurological deficit and underwent posterior decompression and fusion with eventual neurological improvement. ${ }^{31}$ De Peretti et al. ${ }^{20}$ described a case of a male patient who was involved in a motorbike collision with progressive paraplegia and eventually received a diagnosis of an EDH with subsequent evacuation; the patient improved to full neurological recovery. Fitt et al. ${ }^{24}$ described a 68 -year-old male with 24 hours of progressive paraplegia and a minor fall 1 month prior to presentation. The patient was found to have an EDH that was evacuated but with incomplete neurological recovery. Garza-Mercado $^{23}$ described a 32-year-old male who fell after being pushed during an argument and experienced progressive paraplegia and bowel and bladder dysfunction; the patient ultimately underwent decompressive laminectomies and evacuation of an $\mathrm{EDH}$, resulting in a delayed, complete neurological recovery. Even in minor trauma with rapid diagnosis and intervention, outcomes can be catastrophic, as seen in a 63-year-old male who presented after a minor fall and was found to have a C3-T2 EDH that was decompressed; despite surgical intervention, the patient subsequently died..$^{30}$

Most of the patients in our cohort had gradual neurological improvement or neurological preservation after surgical intervention, and no patients had a decline after surgical intervention. However, of the 5 patients in our cohort who had the worst neurological dysfunction prior to surgery (i.e., tetraplegia or paraplegia), only 1 had a significant neurological improvement postoperatively, indicating that early recognition of a compressive hematoma and decompression is essential to prevention of devastating outcomes.

\section{Anticoagulation and Hematoma}

In our series, 8 patients (47\%) were receiving antiplatelet and/or anticoagulant therapy. This was also noted in some patients in our literature review. Elgafy et al. ${ }^{29}$ reported on a 69-year-old male taking the anticoagulant Coumadin who had fallen from a 9-foot height, developed subsequent tetraparesis, and was found to have an EDH and an occult fracture. The patient was treated with decompression without fusion and continued cervical immobilization with improvement in neurological function.

\section{Nontraumatic Etiologies}

There were patients who did not meet our inclusion criteria due to the lack of spinal fractures. We found a series of cases in the literature in which patients with AS underwent epidural anesthesia and sustained a postoperative $\mathrm{EDH}$ requiring urgent decompression. One patient was a 65-year-old male with AS who underwent a laparotomy for abdominal wall reconstruction and had epidural anesthesia with a catheter inserted at the T8-9 level. He was on a preoperative regimen of aspirin for prophylaxis and tinzaparin 


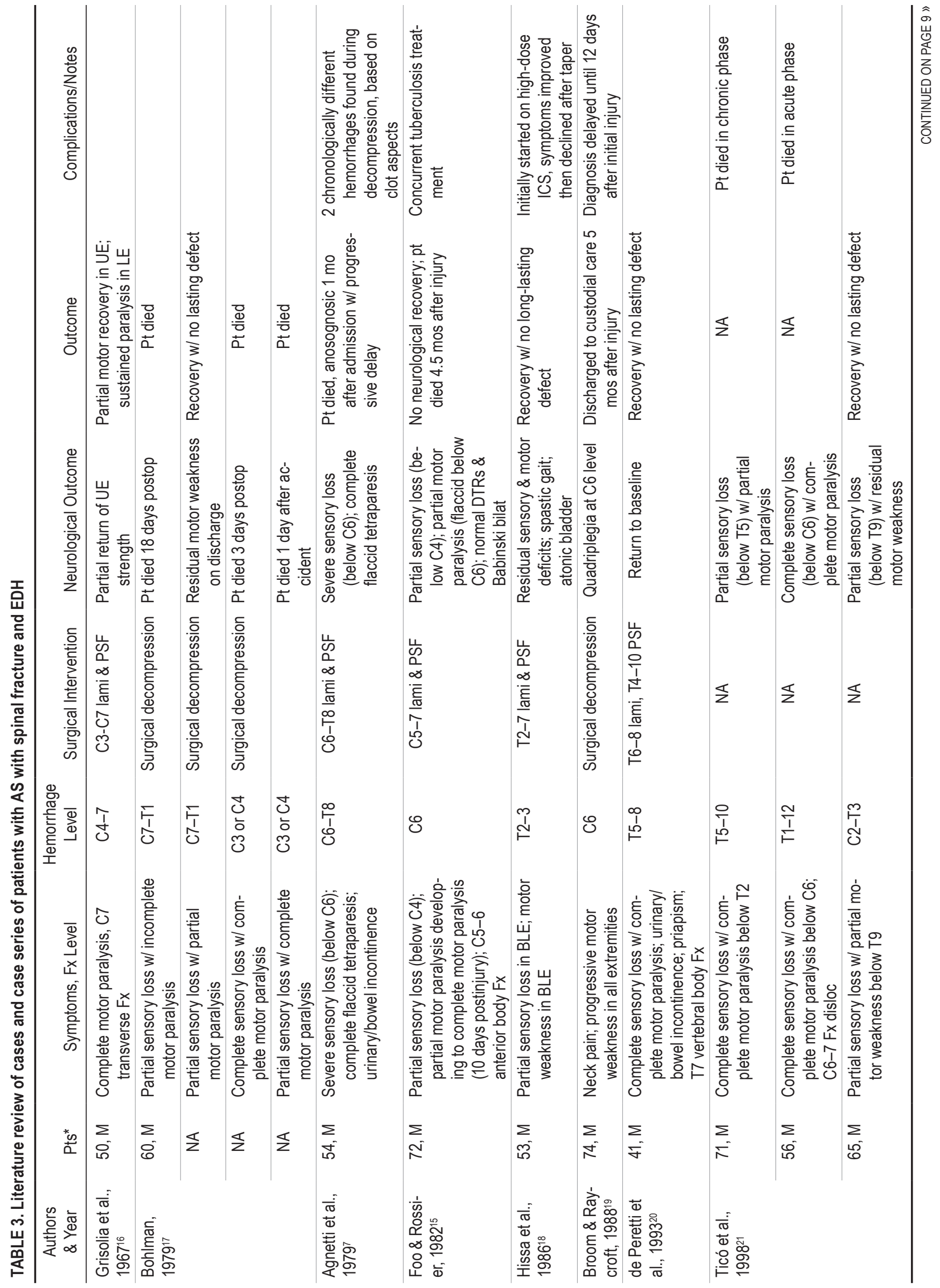




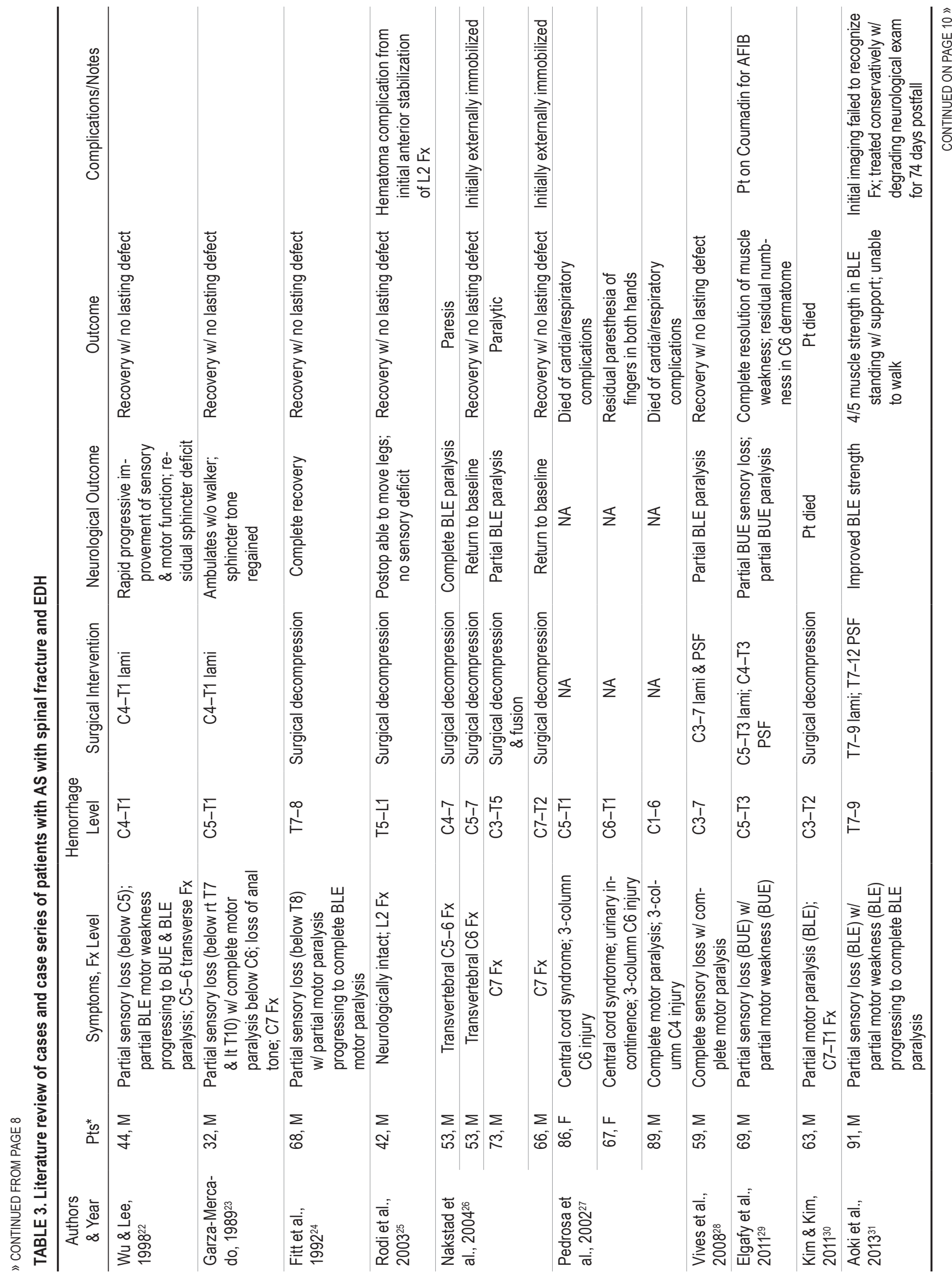




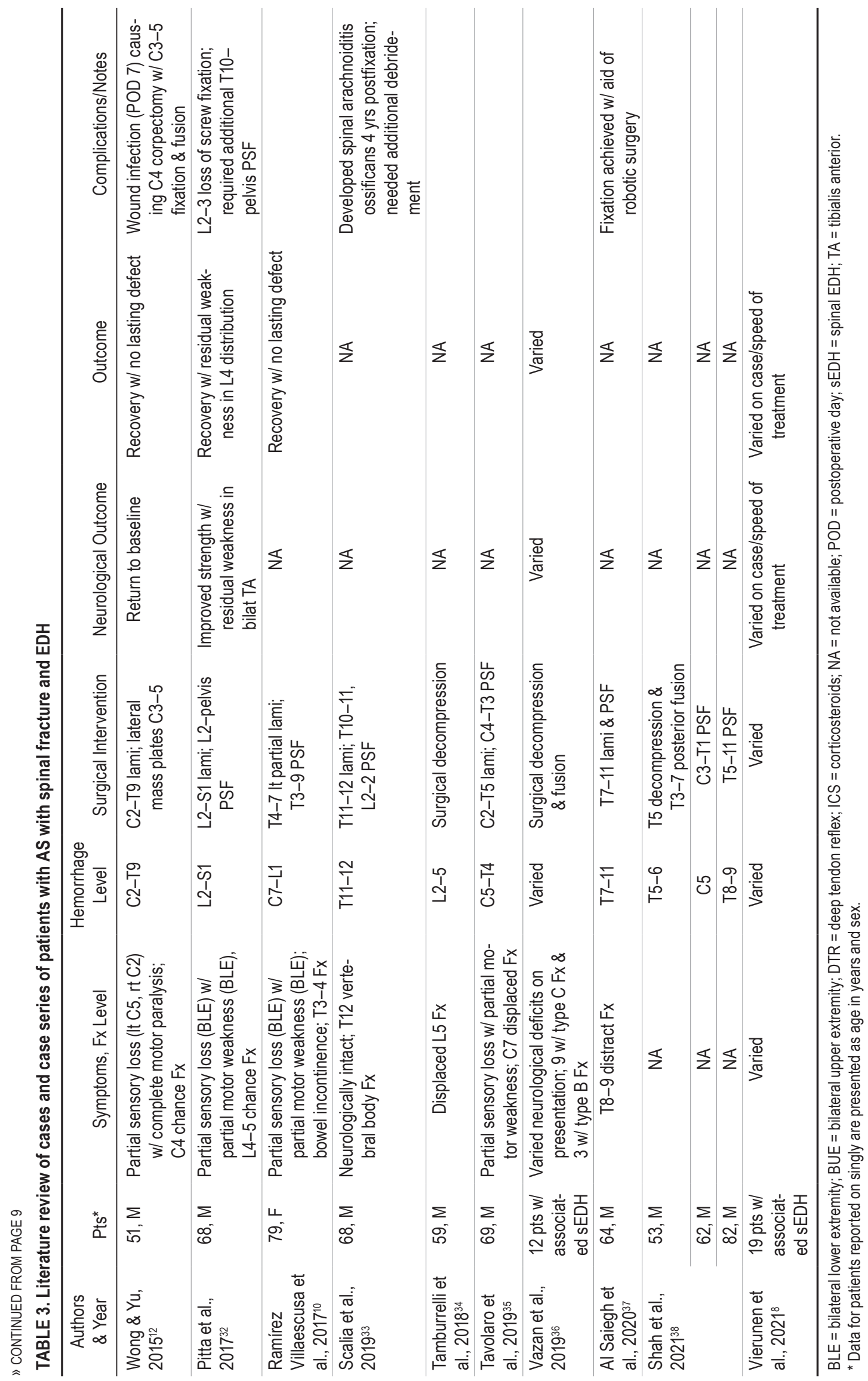


for a previous pulmonary embolism, both of which were discontinued 7 days and 24 hours preoperatively, respectively. The patient woke from surgery with dense hemiparesis, and MRI revealed an EDH from T5 to T11 for which the patient underwent decompressive laminectomies and evacuation. He had minimal neurological recovery with sustained paraplegia after intervention. ${ }^{41}$ Another case reported in the literature is that of a patient with AS who had complete paraplegia 6 days after attempted spinal anesthesia for a total hip arthroplasty that required decompressive laminectomies for EDH, which resulted in eventual motor recovery. ${ }^{42}$ In a large review of 51 patients who developed spinal EDHs after spinal anesthesia, 5 patients had AS. ${ }^{43}$

\section{Spinal Subdural Hematoma}

Although our literature review and cohort focused on EDHs, another closely related entity is spinal subdural hematoma, which can also cause catastrophic neurological injury when there is a delayed diagnosis or without appropriate intervention. A recent study looking at emergency and urgent spinal MRI identified a rate of $18 \%$ of subdural hematoma in traumatic spine fractures. ${ }^{8}$ Esfahani and colleagues ${ }^{44}$ described a 60 -year-old male on a regimen of Coumadin who was getting out of bed when he developed back pain and progressive lower-extremity motor and sensory deficits that required surgical evacuation which eventually resulted in complete neurological recovery. Another patient with AS who presented with a 1-week history of an inability to walk was diagnosed with a spinal subdural hematoma and underwent decompression and, although initially he was able to move his toes, his condition progressed to complete paraplegia. ${ }^{45}$

\section{Study Limitations}

Our intention in this critical literature appraisal and single-institution retrospective review was to emphasize early recognition and intervention for an underappreciated pathology in patients with AS with spinal fractures: EDH that could cause neurological decline or catastrophic neurological injury. Given that this retrospective review constitutes class III evidence (the lowest class of clinical evidence), as are all the other papers cited herein, caution must be taken to ensure that the data reported in our series could underestimate or overestimate the actual incidence of this pathological entity in this population of patients. Further population-wide studies and reviews could help better approximate the true incidence within the literature. However, class I evidence will likely be unavailable due to the ethical considerations in a study that would prevent intervention or investigation of an underlying and potentially devastating pathologic process.

\section{Conclusions}

AS is a relatively rare spinal disorder that leads to a more rigid and fragile spine and predisposes patients to a greater risk of unstable spine fractures that may be occult. Spinal EDHs also have a higher prevalence in this population, and a missed or delayed diagnosis could lead to catastrophic neurological outcomes. Therefore, we present our institutional experience and a literature review to raise awareness and to encourage early MRI or CT myelography studies, even with a low index of suspicion for these lesions. When neurological deficit is present, urgent surgical exploration with decompression is warranted. Early recognition of this relationship is important to ensure that appropriate surgical management includes addressing compression of the neural elements in addition to surgical stabilization.

\section{Acknowledgments}

We acknowledge the Cedars-Sinai Medical Center anesthesiologists Robert Naruse, MD, and Omar Durra, MD, for their expert care in helping us manage these complex patients.

We would like to acknowledge the staff of Deep 6 AI (Pasadena, CA) for their search platform and support, which helped us acquire patient data, and we especially appreciate the explanation of the platform by Mr. Wout Brusselaers, CEO and cofounder of the company.

\section{References}

1. Pedersen SJ, Maksymowych WP. The pathogenesis of ankylosing spondylitis: an update. Curr Rheumatol Rep. 2019; 21(10):58.

2. Daikh DI, Chen PP. Advances in managing ankylosing spondylitis. F1000Prime Rep. 2014;6:78.

3. Braun J, Sieper J. Ankylosing spondylitis. Lancet. 2007; 369(9570):1379-1390.

4. Ruaro B, Casabella A, Molfetta L, Salton F, Confalonieri P, Confalonieri $\mathrm{M}$, et al. What role does trabecular bone score play in chronic inflammatory rheumatic diseases? Front Med (Lausanne). 2020;7:600697.

5. Rustagi T, Drazin D, Oner C, York J, Schroeder GD, Vaccaro $\mathrm{AR}$, et al. Fractures in spinal ankylosing disorders: a narrative review of disease and injury types, treatment techniques, and outcomes. J Orthop Trauma. 2017;31(suppl 4):S57-S74.

6. Leone A, Marino M, Dell'Atti C, Zecchi V, Magarelli N, Colosimo C. Spinal fractures in patients with ankylosing spondylitis. Rheumatol Int. 2016;36(10):1335-1346.

7. Agnetti V, Monaco F, Mutani R. Post-convulsive spinal epidural haematoma in ankylosing spondylitis. Eur Neurol. 1979;18(4):230-233.

8. Vierunen RM, Koivikko MP, Siironen JO, Kerttula LI, Bensch FV. Post-traumatic spinal hematoma in ankylosing spondylitis. Emerg Radiol. 2021;28(3):601-611.

9. Ricart PA, Verma R, Fineberg SJ, Fink KY, Lucas PA, Lo Y, et al. Post-traumatic cervical spine epidural hematoma: incidence and risk factors. Injury. 2017;48(11):2529-2533.

10. Ramírez Villaescusa J, Restrepo Pérez M, Ruiz Picazo D. Spinal epidural hematoma related to vertebral fracture in an atypical rigid diffuse idiopathic skeletal hyperostosis: a case report. Geriatr Orthop Surg Rehabil. 2017;8(1):18-22.

11. Schwake M, Zoubi T, Schipmann S, Stummer W, Ewelt C. Intraoperative myelography of traumatic spine injury in patients with ankylosing spondylitis. Technical note. Acta Neurochir (Wien). 2016;158(4):745-748.

12. Wong AS, Yu DH. Cervical spine fracture in a patient with ankylosing spondylitis causing a C2-T9 spinal epidural hematoma-treatment resulted in a rapid and complete recovery from tetraplegia: case report and literature review. Asian $J$ Neurosurg. 2015;10(1):53.

13. Jacobs WB, Fehlings MG. Ankylosing spondylitis and spinal cord injury: origin, incidence, management, and avoidance. Neurosurg Focus. 2008;24(1):E12.

14. Foo D, Sarkarati M, Marcelino V. Cervical spinal cord injury complicating ankylosing spondylitis. Paraplegia. 1985;23(6): 358-363. 
15. Foo D, Rossier AB. Post-traumatic spinal epidural hematoma. Neurosurgery. 1982;11(1 Pt 1):25-32.

16. Grisolia A, Bell RL, Peltier LF. Fractures and dislocations of the spine complicating ankylosing spondylitis. A report of six cases. J Bone Joint Surg Am. 1967;49(2):339-344.

17. Bohlman HH. Acute fractures and dislocations of the cervical spine. An analysis of three hundred hospitalized patients and review of the literature. J Bone Joint Surg Am. 1979; 61(8):1119-1142.

18. Hissa E, Boumphrey F, Bay J. Spinal epidural hematoma and ankylosing spondylitis. Clin Orthop Relat Res. 1986;(208): 225-227.

19. Broom MJ, Raycroft JF. Complications of fractures of the cervical spine in ankylosing spondylitis. Spine (Phila $\mathrm{Pa}$ 1976). 1988;13(7):763-766.

20. de Peretti F, Hovorka I, Aboulker C, Bonneau G, Argenson C. Fracture of the spine, spinal epidural haematoma and spondylitis. Report of one case and review of the literature. Eur Spine J. 1993;1(4):244-248.

21. Ticó N, Ramon S, Garcia-Ortun F, Ramirez L, Castelló T, Garcia-Fernández L, Lience E. Traumatic spinal cord injury complicating ankylosing spondylitis. Spinal Cord. 1998; 36(5):349-352.

22. Wu CT, Lee ST. Spinal epidural hematoma and ankylosing spondylitis: case report and review of the literature. $J$ Trauma. 1998;44(3):558-561.

23. Garza-Mercado R. Traumatic extradural hematoma of the cervical spine. Neurosurgery. 1989;24(3):410-414.

24. Fitt G, Hennessy O, Thomas D. Case report 709: Transverse fracture with epidural and small paravertebral hematomata, in a patient with ankylosing spondylitis. Skeletal Radiol. 1992;21(1):61-63.

25. Rodi Z, Straus I, Denić K, Deletis V, Vodusek DB. Transient paraplegia revealed by intraoperative neurophysiological monitoring: was it caused by the epidural anesthetic or an epidural hematoma? Anesth Analg. 2003;96(6):1785-1788.

26. Nakstad PH, Server A, Josefsen R. Traumatic cervical injuries in ankylosing spondylitis. Acta Radiol. 2004;45(2): 222-226.

27. Pedrosa I, Jorquera M, Mendez R, Cabeza B. Cervical spine fractures in ankylosing spondylitis: MR findings. Emerg Radiol. 2002:9(1):38-42.

28. Vives MJ, Harris C, Reiter MF, Drzala M. Use of stand-up magnetic resonance imaging for evaluation of a cervicothoracic injury in a patient with ankylosing spondylitis. Spine $J$. 2008;8(4):678-682.

29. Elgafy H, Bransford RJ, Chapman JR. Epidural hematoma associated with occult fracture in ankylosing spondylitis patient: a case report and review of the literature. J Spinal Disord Tech. 2011;24(7):469-473.

30. Kim SW, Kim HS. Clinical images: epidural hematoma in ankylosing spondylitis. Arthritis Rheum. 2011;63(12):3896.

31. Aoki Y, Yamagata M, Ikeda Y, Nakajima F, Nakajima A, Nakagawa K, et al. Failure of conservative treatment for thoracic spine fracture in ankylosing spondylitis: delayed neurological deficit due to spinal epidural hematoma. Mod Rheumatol. 2013;23(5):1008-1012.

32. Pitta M, Wallach CJ, Bauk C, Hamilton WG. Lumbar chance fracture after direct anterior total hip arthroplasty. Arthroplast Today. 2017;3(4):247-250.

33. Scalia G, Certo F, Maione M, Barbagallo GMV. Spinal arachnoiditis ossificans: report of quadruple-triggered case. World Neurosurg. 2019;123:1-6.
34. Tamburrelli FC, Meluzio MC, Masci G, Perna A, Burrofato A, Proietti L. Etiopathogenesis of traumatic spinal epidural hematoma. Neurospine. 2018;15(1):101-107.

35. Tavolaro C, Ghaffar S, Zhou H, Nguyen QT, Bellabarba $\mathrm{C}$, Bransford RJ. Is routine MRI of the spine necessary in trauma patients with ankylosing spinal disorders or is a CT scan sufficient? Spine J. 2019;19(8):1331-1339.

36. Vazan M, Ryang YM, Barz M, Török E, Gempt J, Meyer B. Ankylosing spinal disease-diagnosis and treatment of spine fractures. World Neurosurg. 2019;123:e162-e170.

37. Al Saiegh F, Leibold A, Mouchtouris N, Sabourin V, Stefanelli A, Franco D, et al. Robot-assisted instrumented fusion of a T8-9 extension distraction fracture and epidural hematoma evacuation: 2-dimensional operative video. Oper Neurosurg (Hagerstown). 2020;19(4):E420-E421.

38. Shah NG, Keraliya A, Harris MB, Bono CM, Khurana B. Spinal trauma in DISH and AS: Is MRI essential following the detection of vertebral fractures on CT? Spine J. 2021; 21(4):618-626.

39. Teunissen FR, Verbeek BM, Cha TD, Schwab JH. Spinal cord injury after traumatic spine fracture in patients with ankylosing spinal disorders. J Neurosurg Spine. 2017;27(6): 709-716.

40. Shah NG, Keraliya A, Nunez DB, Schoenfeld A, Harris MB, Bono CM, Khurana B. Injuries to the rigid spine: what the spine surgeon wants to know. Radiographics. 2019;39(2):449466.

41. Anipindi S, Ibrahim N. Epidural haematoma causing paraplegia in a patient with ankylosing spondylitis: a case report. Anesth Pain Med. 2017;7(2):e43873.

42. Li J, Qi K, Zhang Y, Xue C, Xu W. Epidural hematoma after total hip arthroplasty in ankylosing spondylitis patient: a case report and review of the literature. Medicine (Baltimore). 2017;96(19):e6859.

43. Wulf H. Epidural anaesthesia and spinal haematoma. Can J Anaesth. 1996;43(12):1260-1271.

44. Esfahani DR, Shah HP, Behbahani M, Arnone GD, Mehta AI. Spinal subdural hematoma and ankylosing spondylitis: case report and review of literature. Spinal Cord Ser Cases. 2018;4:30.

45. Sokoloff J, Coel MN, Ignelzi RJ. Spinal subdural hematoma. Radiology. 1976;120(1):116.

\section{Disclosures}

Dr. Kim has a Medtronic research grant and is a consultant for Medtronic and DePuy Synthes.

\section{Author Contributions}

Conception and design: Johnson, Hanna. Acquisition of data: Johnson, Hanna, Uddin, Kim. Analysis and interpretation of data: Johnson, Hanna, Uddin, Drazin, Kim. Drafting the article: Johnson, Hanna, Uddin, Drazin, Kim. Critically revising the article: Johnson, Hanna, Uddin, Trontis, Drazin, Kim. Reviewed submitted version of manuscript: all authors. Statistical analysis: Johnson, Hanna, Ross. Administrative/technical/material support Johnson, Hanna, Ross. Study supervision: Johnson, Hanna, Ross.

\section{Correspondence}

J. Patrick Johnson: Cedars-Sinai Medical Center, Los Angeles, CA.patrick.johnson@cshs.org. 